\title{
Gated-SPECT myocardial perfusion imaging in women after acute coronary syndrome
}

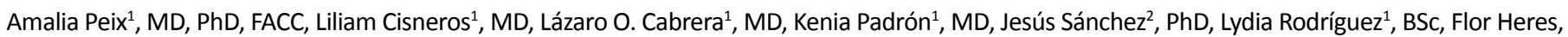
$\mathrm{MD}^{1}$, Sheila Hechevarría ${ }^{1}, \mathrm{MD}$, Elida Batista ${ }^{1}, \mathrm{MD}$

Peix A, Cisneros L, Cabrera LO, et al. Gated-SPECT myocardial perfusion imaging in women after acute coronary syndrome. Curr Res Cardiol 2017;4(2):24-28.

OBJECTIVES: To assess the prognostic value of gated-SPECT myocardial perfusion imaging (MPI) in women after acute coronary syndrome (ACS), as well as to evaluate whether the intraventricular dyssynchrony shows any difference in women according to the type of ACS.

METHODS: Ninety women (mean age: $58 \pm 9$ years) admitted between April 2011 and April 2014 with diagnosis of ACS were included. They were divided in two groups: those with ST elevation myocardial infarction (STEMI) (group 1, 54 patients), and those with non-ST elevation myocardial infarction (NSTEMI) (group 2, 36 patients). All underwent a gated-SPECT two-day MPI with a stress rest protocol. A one year clinical follow-up was done.

RESULTS: Summed stress (SSS) and rest scores (SRS) were significantly greater in STEMI patients (11.33 vs. 4.86 for SSS, and 8.24 vs. 2.66 for SRS). Left ventricular ejection fraction was significantly higher in those with NSTEMI (both post-stress: $68.75 \%$ vs. $58.54 \%$ in STEMI, and at rest: $67.22 \%$ vs. $58.75 \%$ ). Phase-derived standard deviation and histogram bandwidth were significantly different between both groups. STEMI patients showed a more asynchronous behavior. Fourteen adverse cardiac events appeared during follow-up. SSS and the percentage of maximal heart rate (MHR) achieved during maximal stress were associated with the occurrence of cardiac events in NSTEMI patients, but not in those with STEMI.

CONCLUSION: A gated-SPECT MPI can be useful to risk stratifies women after ACS. SSS and percentage of MHR were the best predictors of adverse cardiac events among NSTEMI patients. STEMI patients showed a more asynchronous behavior.

Key Words: Myocardial perfusion imaging; Acute coronary syndrome; Women; Gated-SPECT; Intraventricular synchronism
Coronary artery disease (CAD) is the leading cause of mortality worldwide. Thanks to the advances in diagnosis and management of the acute coronary syndrome (ACS), one of the most severe forms of presentation of CAD, the cardiovascular mortality in men has been reduced over the past decade. However, the mortality rate among women has continued to increase (1). One of the reasons advocated is the fact that women with ACS are less likely to undergo diagnostic and therapeutic procedures compared to men (2).

Patients with a previous ACS have a high risk of developing adverse cardiac events during the first year following the acute episode. Thus, adequate risk stratification is of crucial importance for a patient-tailored treatment.

The extension of myocardium at risk and the left ventricular function are two of the most important factors which determine prognosis in patients with CAD. Gated-SPECT myocardial perfusion imaging (MPI) allows measuring both. It has been shown that the post-stress left ventricular ejection fraction (LVEF) and end-diastolic ventricular volume significantly increase the prognostic power to predict hard cardiac events, compared to clinical data, exercise variables, LVEF at rest and perfusion status. Event-specific survival analysis considering perfusion and function variables has shown that, although the perfusion abnormalities constitute the best predictor of myocardial infarction (MI), the post-stress LVEF is the best predictor of cardiac death (3-6). However, as far as we know, there is no paper published on post-ACS imaging for risk stratification in women.

Another interesting aspect which can be assessed by using phase analysis in gated-SPECT imaging is the left intraventricular synchronism $(7,8)$. LV synchronicity is significantly impaired soon after acute myocardial infarction (AMI), and LV dyssynchrony is related to LVEF and has an additional detrimental effect on LV function, beyond infarct size and the anterior location of AMI (9). For Murrow et al. (10) the improvement in mechanical dyssynchrony after myocardial infarction correlates with beneficial ventricular remodeling. Nevertheless, a gap in the knowledge about the value of intraventricular dyssynchrony in women after ACS still remains.

Nowadays, risk stratification after an ACS depends mainly on clinical, electrocardiographic and angiographic variables, but this approach has some limitations. We hypothesized that the extension of the perfusion defect by gated-SPECT MPI and the presence of intraventricular dyssynchrony can contribute to risk stratify women after ACS. The aim of this study was then, in the first place, to assess the prognostic value of gated-SPECT MPI at medium-term in women after ACS and second, to evaluate whether the intraventricular dyssynchrony shows any difference in women according to the type of ACS.

\section{MATERIALS AND METHODS}

\section{Study population}

We studied 90 female patients (mean age: $58 \pm 9$ years), who were referred by their attending physicians to the Nuclear Medicine Department of the Institute of Cardiology from April 2011 to April 2014 with the following inclusion criteria: 25 years of age and older, with diagnosis of ACS prior to the MPI (mean: 2 months previously), able to exercise on treadmill or bicycle. Exclusion criteria were: pregnancy, previous coronary artery bypass graft $(\mathrm{CABG})$ or any open-heart surgery, significant valvular disease, nonischemic dilated cardiomyopathy, left ventricular bundle branch block (LBBB), arrhythmias which prevent the gated acquisition, or pacemaker implanted before the nuclear test.

Regarding coronary risk factors, there were considered as follows: diabetes mellitus: fasting blood glucose $\geq 5.55 \mathrm{mmol} / \mathrm{L}(\geq 100 \mathrm{mg} / \mathrm{dL}$ ) or treatment for diabetes; dyslipidemia: fasting plasma triglycerides $\geq 1.70 \mathrm{mmol} / \mathrm{L}(\geq 150$ $\mathrm{mg} / \mathrm{dL}$ ) and/or HDL cholesterol $<1.29 \mathrm{mmol} / \mathrm{L}(<50 \mathrm{mg} / \mathrm{dL}$ ); high blood pressure: systolic blood pressure $\geq 130 \mathrm{mmHg}$ or diastolic $\geq 85 \mathrm{mmHg}$ or anti-hypertensive treatment; smoking habit: current smokers in the year before the ACS.

These patients were divided in two groups: those with ST elevation myocardial infarction (STEMI) (group 1, 54 patients), and those with non-ST elevation myocardial infarction (NSTEMI) (group 2, 36 patients). Each patient underwent a $99 \mathrm{~m}$-technetium methoxy-isobutyl-isonitrile (99mTc-MIBI) gated-SPECT MPI, following a two-day protocol: exercise stress/rest, including left ventricular dyssynchrony assessment by phase analysis. A one year clinical follow-up (FU) was done to register the occurrence of adverse cardiac events (unstable angina, non-fatal myocardial infarction, arrhythmias potentially lethal, percutaneous coronary intervention -PCI, CABG, or death).

${ }^{1}$ Institute of Cardiology, La Habana, Cuba, UK, ${ }^{2}$ Institute of Cybernetics, Mathematics and Physics, La Habana, Cuba

Correspondence: Dr Amalia Peix, MD, PhD, Nuclear Medicine Department, Institute of Cardiology, 17 No. 702, Vedado. CP 10 400, La Habana, Cuba, Telephone 537 838 6080,E-mail peix@infomed.sld.cu

Received: September 09, 2016, Accepted: July 18, 2017, Published: July 20, 2017

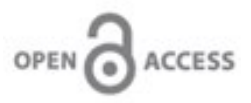

This open-access article is distributed under the terms of the Creative Commons Attribution Non-Commercial License (CC BY-NC) (http:// creativecommons.org/licenses/by-nc/4.0/), which permits reuse, distribution and reproduction of the article, provided that the original work is properly cited and the reuse is restricted to noncommercial purposes. For commercial reuse, contact reprints@pulsus.com

Curr Res Cardiol Vol 4 No 2 Summer 2017 
This study complies with the Declaration of Helsinki. The review board of the Institute of Cardiology approved the study, and written informed consent was obtained from all patients.

\section{Gated-SPECT MPI}

The first day of the study all patients underwent a symptom-limited treadmill exercise stress test (MTM-1 500 med, Schiller, Switzerland) following the Bruce protocol. At peak exercise, a dose of $740 \mathrm{MBq}$ of $99 \mathrm{mTc}$-MIBI was administered intravenously, and the patient continued to exercise for an additional period of $60-90$ seconds when possible. Post-stress images were acquired at 60 minutes after tracer injection, using a rotating dual-head gamma camera (Nucline Spirit DHV, Mediso, Hungary) equipped with lowenergy, high-resolution, parallel-hole collimators, with a $20 \%$ energy window centred on the $140 \mathrm{keV}$ photopeak. Sixty-four projections (20 seconds per projection), eight frames/cycle, with a $64 \times 64$ matrix were obtained over a 1800 orbit. The following day, rest images were acquired at 60 minutes after the intravenous injection of $740 \mathrm{MBq}$ of $99 \mathrm{mTc}-\mathrm{MIBI}$. Imaging was always performed in a supine position.

SPECT images were reconstructed using filtered back-projection with a Butterworth filter, order 7 and a cut-off frequency of 0.25 cycles/pixel. No attenuation or scatter correction was applied. All patients were studied 72 hours after the withdrawal of cardiovascular medication.

\section{SPECT image interpretation}

Semi-quantitative visual interpretation of images employed short-axis and vertical long-axis tomograms divided into 17 segments (11). Each segment was scored by the consensus of two expert independent observers who were unaware of the clinical and angiographic data, using a five-point scoring system (from $0=$ normal to $4=$ absence of myocardial uptake). Disagreements, including any score in each SPECT segment were resolved by consensus. Segments with reduced tracer uptake were considered to be reversible defects if the score decreased $\geq 1$ point from stress to rest. Summed stress, summed rest and summed difference scores (SSS, SRS and SDS) were obtained. If the summed difference score was 4 or greater it was considered as presence of stress-induced ischemia.

The assessment of regional wall motion (WM) was performed by visual inspection of gated tomograms in cine mode for semi-quantitative scoring. The LV myocardium was divided into 17 segments. Segmental WM was classified as: normal, hypokinesis, akinesis or dyskinesis. An operatorindependent analysis of regional WM and LVEF was made using dedicated software (Emory Cardiac Toolbox -ECTb-, Syntermed, Inc., Atlanta, Georgia, USA). The difference between LVEF from post-stress and rest acquisitions was defined as Delta LVEF (Delta LVEF = post-stress LVEF rest LVEF). The left intraventricular mechanical dyssynchrony was evaluated at rest and post-stress by using the phase analysis of the gated SPECT MPI included in the ECTb, previously described (12)

\section{Statistical analysis}

Categorical variables are expressed as numbers and percentages, and compared when necessary with the chi-square test and the Fisher exact test. Continuous variables are expressed as mean \pm standard deviation (SD), and the non-parametric Kolmogorov-Smirnov normality test (K-S test) was applied to check variables normality. For independent observations, the nonpaired Student t- test, the Mann-Whitney U test or the Kruskal-Wallis test were applied. For paired observations, the paired Student $\mathrm{t}$ - test was used. Odds ratios (ORs) were calculated with $95 \%$ confidence intervals $(\mathrm{CI})$ by using the logistic regression method, as estimates of the risk associated with a particular variable. A value of $\mathrm{p}<0.05$ was considered significant.

\section{RESULTS}

\section{Patient characteristics}

Clinical characteristics of patients are shown in Table 1.

There were no significant differences between both groups regarding age and presence of coronary risk factors, except the family history of CAD, which was significantly more frequent in NSTEMI patients. More STEMI patients were postmenopausal, but the difference was not significant. Forty-four percent of postmenopausal patients among those with STEMI had 10 years or more of post-menopause, vs. $36 \%$ of postmenopausal patients among those with NSTEMI. Only the smoking habit showed a significant difference according to the postmenopausal status (more non-menopausal and with less than 10 years of post-menopause smoked, $p=0.01$ ). All diabetic patients were type 2 , with a disease duration of $5 \pm 1$ years prior to the ACS. There were no
TABLE 1

\section{Patient characteristics}

\begin{tabular}{cccc}
\hline & $\begin{array}{c}\text { Group 1 - STEMI } \\
(\mathrm{n}=54)\end{array}$ & $\begin{array}{c}\text { Group 2 - NSTEMI } \\
(\mathrm{n}=36)\end{array}$ & $\mathrm{p}$ \\
\hline Age (years) & $61 \pm 8$ & $62 \pm 8$ & $\mathrm{NS}$ \\
Presence of chest pain & $28(52 \%)$ & $30(83 \%)$ & 0.004 \\
DM & $14(26 \%)$ & $10(28 \%)$ & $\mathrm{NS}$ \\
High Blood Pressure & $37(69 \%)$ & $29(80 \%)$ & $\mathrm{NS}$ \\
Dyslipidemia & $21(39 \%)$ & $12(33 \%)$ & $\mathrm{NS}$ \\
Smoking & $25(46 \%)$ & $17(47 \%)$ & $\mathrm{NS}$ \\
Family history of CAD & $10(19 \%)$ & $16(44 \%)$ & 0.008 \\
Postmenopause & $46(85 \%)$ & $22(61 \%)$ & $\mathrm{NS}$ \\
\hline
\end{tabular}

significant differences regarding the rest of coronary risk factors.

No significant differences were found regarding the post-MI risk factors control between both groups.

Chest pain as symptom referred by the patients before the gated-SPECT was significantly more frequent among NSTEMI patients: $83 \%$ vs. $52 \%$ among those with STEMI, $\mathrm{p}=0.004$. Patients from both groups experienced more typical than atypical chest pain ( $37 \%$ vs. $15 \%$ among STEMI, and $58 \%$ vs. $25 \%$ among NSTEMI).

\section{Stress results}

Stress characteristics are presented in Table 2.

There was no significant difference between both groups. Chest pain and ST depression were slightly more frequent in STEMI patients, but the difference was not significant. In four STEMI patients the ST depression coincided with chest pain, the rest were silent. Among NSTEMI patients only one showed ST depression with chest pain. Arrhythmias during stress appeared in $12(22 \%)$ patients among STEMI patients, while in $7(19 \%)$ among those with NSTEMI, p NS.

\section{Gated-SPECT MPI}

\section{Myocardial perfusion and left ventricular function}

Both SSS and SRS were significantly greater in STEMI patients than in NSTEMI: $11.33 \pm 7.94$ vs. $4.86 \pm 6.24$ for SSS, $p<0.0001$, as well as 8.24 \pm 7.20 vs. $2.66 \pm 3.78$ for SRS, $p<0.0001$. SDS did not show a significant difference between both groups: $3.09 \pm 4.41$ in STEMI vs. $2.19 \pm 4.76$ in NSTEMI. The mean transient ischemic dilatation was the same for both groups: $1.04 \pm 0.16$ (STEMI) and $1.04 \pm 0.13$ (NSTEMI).

Left ventricular ejection fraction was significantly higher in those with NSTEMI (post-stress LVEF: $68.75 \pm 5.22 \%$ vs. $58.54 \pm 14.44 \%$ in STEMI, $\mathrm{p}=0.0001$, as well as rest LVEF: $67.22 \pm 6.05 \%$ in NSTEMI vs. $58.75 \pm$ $15.03 \%$ in STEMI, $p=0.003$ ). Delta LVEF was significantly different, with STEMI patients showing more post-stress LVEF reduction than those with NSTEMI: $-1.33 \%$ vs. $1.94 \%$, $p=0.02$.

\section{Intraventricular synchronism}

Both intra-ventricular synchronism parameters: Phase-derived standard deviation (SD) and histogram bandwidth (HBW) were significant different between both groups, both at rest and post-stress. STEMI patients showed the more asynchronous behavior (Figure 1).

Rest phase SD values were: $37.75 \pm 20.88$ degrees (STEMI) vs. $19.36 \pm 8.15$ degrees (NSTEMI), $\mathrm{p}=0.0001$. Post-stress values were: $35.37 \pm 20.75$ degrees (STEMI) vs. $22.93 \pm 15.21$ degrees (NSTEMI), $p=0.003$. Rest $\mathrm{HBW}$ values were: $113.29 \pm 80.02$ degrees (STEMI) vs. $51.89 \pm 18.40$ degrees (NSTEMI), $\mathrm{p}=0.0001$. Post-stress values were: $104.60 \pm 69.99$ degrees (STEMI) vs. 59.87 \pm 40.55 degrees (NSTEMI), $\mathrm{p}=0.001$. There were no significant differences between rest and post-stress values, neither for phase SD nor for HBW.

\section{Follow-up}

Fourteen adverse cardiac events appeared during the FU: seven (13\%) in patients with STEMI, and seven (19\%) among those with NSTEMI, pNS.

Among STEMI patients, the following events appeared: one non-fatal myocardial infarction, one admission for unstable angina, one episode of ventricular tachycardia with ulterior implantable cardiac defibrillator, three PCI and one CABG. NSTEMI patients had: six admissions for unstable angina and one cardiac death. 
TABLE 2

\section{Stress results}

\begin{tabular}{cccc}
\hline & $\begin{array}{c}\text { Group 1 - } \\
\text { STEMI }(\mathbf{n = 5 4})\end{array}$ & $\begin{array}{c}\text { Group 2 - NSTEMI } \\
(\mathbf{n}=\mathbf{3 6})\end{array}$ & $\mathbf{p}$ \\
\hline Exercise duration $(\mathrm{min})$ & $6 \pm 1$ & $7 \pm 2$ & NS \\
METS & $4.71 \pm 1.38$ & $5.13 \pm 1.30$ & NS \\
\%MHR achieved & 927 & $93 \pm 6$ & NS \\
Chest pain at stress & $9(17 \%)$ & $4(11 \%)$ & NS \\
ST depression & $9(17 \%)$ & $2(6 \%)$ & NS \\
Peak systolic BP & 16623 & $170 \pm 17$ & NS \\
Peak diastolic BP & $97 \pm 12$ & $96 \pm 10$ & NS \\
\hline
\end{tabular}

SSS and the percentage of maximal heart rate (MHR) achieved during maximal stress were associated with the occurrence of cardiac events in NSTEMI patients, but not in those with STEMI. Among NSTEMI patients, the $42.86 \%$ of patients who developed any adverse cardiac event during the FU achieved less than $85 \%$ MHR during stress, vs. $3.45 \%$ of those who did not have cardiac events (OR: 21, $\mathrm{p}=0.02$, 95\% CI: 1.58-278.11). Regarding the SSS, the $57.14 \%$ of patients who developed cardiac events had a moderate to severe ischemia, vs. $13.79 \%$ of those who did not have events (OR: 8.33 , $\mathrm{p}=0.03,95 \%$ CI: $1.24-55.67$ ).

\section{DISCUSSION}

In the present work, summed stress and rest scores, LVEF, phase SD and HBW were significantly different between STEMI and NSTEMI groups. STEMI patients showed greater perfusion defects, a more compromised ventricular function and were more dyssynchronous than the NSTEMI ones. This is a logical behavior considering that STEMI patients have more extensive myocardial damage, but as far as we know, there are no previous reports of the synchronism behavior after ACS specifically in women. This can be considered as the novelty of this work, mainly taking into account that the differences between STEMI and NSTEMI ACS presentations are analyzed.

Regarding the specificities of the risk factors profile in women, it is interesting to point out that although postmenopause poses a well-known risk for developing CAD in women, probably due to the loss of the protective effects of estrogens, with elevated total cholesterol, LDL cholesterol, and triglyceride levels, as well as lower HDL-C levels and endothelial dysfunction (13-15), in our sample the fact of having a $\geq 10$-year postmenopausal period was not associated to a worst coronary risk profile.

By the contrary, the only risk factor with a significant difference, the smoking habit, was more frequent in younger women. This is according to the results of Otten et al (16), who in a group of 1755 women and 4991 men with STEMI treated with primary PCI, found a higher percentage of current smokers in younger women (67\%) in comparison with men (60\%). Smoking increases the risk of an AMI relatively more in young women than in young men $(17,18)$, by increasing oxidative stress and endothelial dysfunction (19), which counteracts the protective vasodilating effects of endogenous estrogens in women before menopause.

\section{Myocardial perfusion and left ventricular function}

As far as we know, there is no published data on the comparison of perfusion, function and intraventricular synchronism between women with STEMI and NSTEMI. Our STEMI patients showed greater perfusion defects and a more compromised ventricular function than the NSTEMI ones, which seems logical taking into account that STEMI patients are more severely diseased, with a bigger and transmural area of myocardial infarction. Even post-stress LVEF reduction was significantly higher in STEMI patients, which can be considered as a sign of myocardial stunning $(20,21)$.

It is well known that SSS $>8, \geq 10 \%$ of abnormal myocardium, $\geq 10 \%$ of ischemic myocardium, as well as post-stress LVEF lower than rest LVEF, are features that can be considered as high-risk indicators of future cardiac adverse events $(22,23)$. Thus, it seems that these same features can also help to risk stratify female patients after ACS.

\section{Intraventricular synchronism}

Asynchronous motion often appears in patients with myocardial infarction and has been associated with infarct size (24) and left ventricular remodeling at six-month FU (25).

Experimental and clinical reports have demonstrated that dyssynchrony results in decreased cardiac output, slowed relaxation rates, reduced peak filling velocity, and increased myocardial energy demand (26,27), and may be associated with abnormalities of myocardial perfusion $(28,29)$. It has been suggested that abnormal patterns of contraction and myocardial stretch are likely to increase mechanical loading and myocardial work, which could impair functional recovery after ischemic injury (30).

According to this, our results showed that patients with STEMI, with more abnormal contraction patterns due to the presence of transmural myocardial infarction, had the more asynchronous behavior both at rest and post-stress, considering both phase variables (phase SD and HBW).

We did not find any significant difference between rest and post-stress values, neither for phase SD nor for HBW. This fact deserves some explanation: for this study we acquired images at one hour post-stress and it is known that ventricular function reflects the real-time behavior (that is, the moment of acquisition) so, the ischemia-induced contractile changes are no longer present in the vast majority of cases at one hour post-stress. However, if the acquisition is done between 10 and 30 minutes, either by using thallium-201 (31), or with technetium-labeled compounds at 15-30 minutes post-stress (32), it is possible to find ischemic-induced changes in the synchronism variables.

For Hida et al. (32), the mechanisms responsible for dyssynchrony are the temporal contraction delay (as in LBBB), and the heterogeneous contraction due to fibrosis and ischemia, which could explain our results. Other authors, such as Haugaa et al. (33), also suggest that the mechanical dispersion of the contraction may be a representation of the scar tissue scatter within the myocardium, as well as a substrate for arrhythmia and sudden cardiac death.

Although in our patients we did not find any significant relationship between synchronism status and adverse cardiac events, this is a topic which needs more research with bigger samples, probably considering some cut-off values, because the fact of having values of SD and HBW only above the normal limits does not necessarily implies a clinical significance.

\section{Follow-up}

In a review of individual patient-level data from three established ACS registries published by Bugiardini et al (34), despite presenting with higher risk characteristics and having higher in-hospital and six months risk of death, women with ACS and obstructive CAD were apparently treated less aggressively with secondary preventive drugs than were men, being less likely to receive aspirin, beta-blockers and statins at discharge. Overall, coronary revascularization appears to be performed in a similar proportion of women and men, once angiography has been performed and the coronary anatomy is known. Nevertheless, substantial geographic variation exists in the relative rate of coronary angiography in men and women. On the other hand, Berger et al. (35), who analyzed data from 11 ACS trials (including 136,247 patients, $28 \%$ women), found that there are sex-based differences in 30-day mortality among ACS patients which vary depending on clinical presentation. However, these differences are markedly attenuated following adjustment for clinical differences and angiographic data. Similarly, Berthillot et al. (36), in a study of 479 consecutive patients, $28 \%$ women, did not report any gender difference for in-hospital adverse events in patients treated invasively for a NSTEMI.

Thus, these results are somehow controversial and it is understandable that diagnostic tests with appropriate sensitivity for risk stratification are required.

By assessing the extent and severity of defect size and its degree of reversibility, SPECT-MPI provided a continuum of risk stratification $(37,38)$. Cerci et al. (39), in 2225 women referred for SPECT-MPI who were followed by $3.7 \pm$ 1.4 years, found that SPECT-MPI results had a 3.02 times higher incidence of all-cause death during the FU period, when compared with women with normal results.

However, among our patients, although there was no significant difference in the number of adverse events according to the ACS type, SSS and the percentage of maximal heart rate (MHR) achieved during maximal stress were the only variables associated with the occurrence of cardiac events in NSTEMI patients, but not in those with STEMI, and we do not have explanation for these behavior, except the possible influence of our small sample. Among NSTEMI patients, the $42.86 \%$ of patients who developed any adverse cardiac event during the FU achieved less than 85\% MHR during stress, vs. $3.45 \%$ of those who did not have cardiac events (OR: 21 , $\mathrm{p}=0.02,95 \%$ CI: $1.58-278.11)$.

Patients who achieved a reduced percentage of MHR during the stress show a worst functional capacity, and it is well known that less than 5 METS achieved constitute a variable of bad prognosis. 

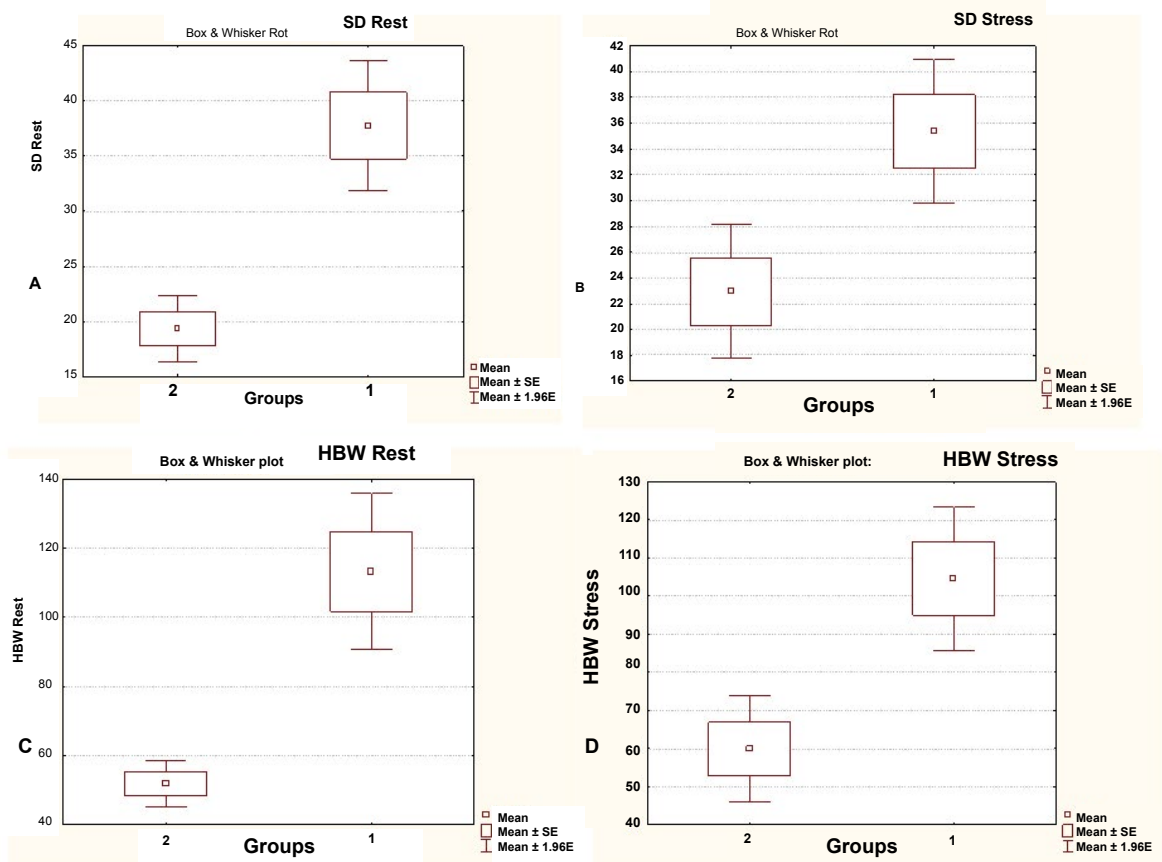

Figure 1) $t$-Test comparison between Group 1(STEMI) and Group 2 (NSTEMI) for synchronism parameters. A) Phase standard deviation (SD) at rest, $p$-value $=0.000029$. B) Phase standard deviation (SD) at stress, p-value $=0.0037$. C) Histogram bandwidth (HBW) at rest, p-value $=0.000155$. D) Histogram bandwidth (HBW) at stress, $p-$ value $=0.001274$. The four parameters were significantly different between STEMI and NSTEMI patients

Regarding the SSS, the $57.14 \%$ of patients who developed cardiac events had a moderate to severe ischemia, vs. $13.79 \%$ of those who did not have events (OR: $8.33, p=0.03,95 \%$ CI: $1.24-55.67$ )

With reference to the intraventricular synchronism, although for some authors (40-42), both dyssynchrony or stress-induced impairment of dyssynchrony are independent predictors of mortality, and we found a difference among our STEMI and NSTEMI patients, probably due again to the small sample we included, no predictive variables of adverse events were identified.

\section{Limitations}

The sample size is small and therefore so being the number of deaths and other adverse cardiac events. A longer follow-up period would have provided more information, so this research can be considered as hypothesis generating. A second work comparing women and women for risk stratification after ACS is under preparation.

\section{CONCLUSION}

A gated-SPECT MPI can be useful to risk stratify women after an acute coronary syndrome. SSS and percentage of MHR were the best predictors of adverse cardiac events among NSTEMI patients. STEMI patients showed the more asynchronous behavior.

\section{ACKNOWLEDGMENT}

We are grateful to Adrienne Hunter, Ph.D., for her patience and dedication in reviewing the manuscript.

\section{CONFLICT OF INTEREST}

None.

\section{REFERENCES}

1. American Heart Association. Heart Disease and Stroke Statistics- 2004 Update. Dallas, TX: American Heart Association, 2003.

2. El-Menyar A, Ahmed E, Albinali $\mathrm{H}$, et al. Mortality trends in women and men presenting with acute coronary syndrome: Insights from a 20-year registry. PLOS ONE 2013;8:1-10

3. Pollock SG, Abbott RD, Boucher CA, et al. Independent and incremental prognostic value of tests performed in hierarchical order to evaluate patients with suspected coronary artery disease. Circulation 1992;85:237-48.

4. Berman DS, Hachamovitch $\mathrm{R}$, Kiat $\mathrm{H}$, et al. Incremental value of prognostic testing in patients with known or suspected ischemic heart disease: a basis for optimal utilization of exercise technetium- $99 \mathrm{~m}$ sestamibi myocardial perfusion single-photon emission computed tomography. J Am Coll Cardiol 1995;26:639-47.

5. Hachamovitch R, Berman DS, Kiat H, et al. Exercise myocardial perfusion SPECT in patients without known coronary artery disease: incremental prognostic, value and impact on subsequent patient management. Circulation 1996;93:905-14.

6. Hachamovitch R, Berman DS, Shaw LJ, et al. Incremental prognostic value of myocardial perfusion single photon emission computed tomography for the prediction of cardiac death. Circulation 1998;97:535-43.

7. Aljaroudi W, Koneru J, Heo J, et al. Impact of ischemia on left ventricular dyssynchrony by phase analysis of gated single photon emission computed tomography myocardial perfusion imaging. J Nucl Cardiol 2011;18:36-2.

8. Samad Z, Atchley A, Trimble M, et al. Prevalence and predictors of mechanical dyssynchrony as defined by phase analysis in patients with left ventricular dysfunction undergoing gated SPECT myocardial perfusion imaging. J Nucl Cardiol 2011;18:24-0.

9. Nucifora G, Bertini M, Ajmone MM, et al. Impact of left ventricular dyssynchrony early on left ventricular function after first acute myocardial infarction. Am J Cardiol 2010;105:306-11.

10. Murrow J, Esteves F, Galt J, et al. Characterization of mechanical dyssynchrony measured by gated single photon emission computed tomography phase analysis after acute ST-elevation myocardial infarction. J Nucl Cardiol 2011;18:912-19.

11. Cerqueira M, Weissman M, Dilsizian V, et al. Standardized myocardial segmentation and nomenclature for tomographic imaging of the heart. Circulation 2002;105:539-42.

12. Chen J, Garcia E, Folks R, et al. Onset of left ventricular mechanical contraction as determined by phase analysis of ECG-gated myocardial perfusion SPECT imaging: Developing of a diagnostic tool for assessment of cardiac mechanical dyssynchrony. J Nucl Cardiol 2005;12:687-95.

13. Jneid $\mathrm{H}$, Thacker HL. Coronary artery disease in women: different, often undertreated. Cleve Clin J Med 2001;68:441-48.

14. Coca A, Cea-Calvo L, Lozano JV, et al. High-density lipoprotein cholesterol and cardiovascular disease in Spanish hypertensive women. The RIMHA study. Rev Esp Cardiol 2009;62:1022-31.

15. Mendelsohn ME, Karas RH. Molecular and cellular basis of cardiovascular gender differences. Science 2005;308:1583-87. 
16. Otten A, Maas A, Ottervanger JP, et al. Is the difference in outcome between men and women treated by primary percutaneous coronary intervention age dependent? Gender difference in STEMI stratified on age. Eur Heart J Acute Cardiovasc Care 2013;2:334-41.

17. Prescott E, Hippe M, Schnohr P, et al. Smoking and risk of myocardial infarction in women and men: longitudinal population study. BMJ $1998 ; 316: 1043-47$

18. Grundtvig M, Hagen TP, German M, et al. Sex-based differences in premature first myocardial infarction caused by smoking: twice as many years lost by women as by men. Eur J Cardiovasc Prev Rehabil 2009; 16:174-79.

19. Barbieri SS, Zacchi E, Amadio P, et al. Cytokines present in smokers' serum interact with smoke components to enhance endothelial dysfunction. Cardiovascular Research 2011;90:475-83.

20. Mut F, Beretta M, Vidal I. Identification of myocardial stunning by means of gated perfusion SPECT in patients undergoing ischaemic stress myocardial tests. World J Nucl Med 2003;2:122-25.

21. Mut F, Giubbini R, Vitola J, et al. Early post-exercise acquisition of $99 \mathrm{mTc}$-sestamibi gated SPECT to detect myocardial stunning: results of an IAEA multicentre study. J Nucl Cardiol 2014;21:1168-76.

22. Mieres J, Gulati M, Bairey MN, et al. Role of noninvasive testing in the clinical evaluation of women with suspected ischemic heart disease. A consensus statement From the American Heart Association. Circulation 2014;130:350-79.

23. Nuclear Cardiology: Guidance and recommendations for implementation in developing countries (IAEA Human Health Series, No. 23). Viena, 2012.

24.Zhang Y, Chan AK, Yu CM, et al. Left ventricular systolic asynchrony after acute myocardial infarction in patients with narrow QRS complexes. Am Heart J 2005;149:497-03.

25. Mollema SA, Liem SS, Suffoletto MS, et al. Left ventricular dyssynchrony acutely after myocardial infarction predicts left ventricular remodeling. J Am Coll Cardiol 2007;50:1532-40.

26.Zile MR, Blaustein AS, Shimizu G, et al. Right ventricular pacing reduces the rate of left ventricular relaxation and filling. J Am Coll Cardiol 1987; 10:702-09.

27.Takeuchi M, Fujitani K, Kurogane K, et al. Effects of left ventricular asynchrony on time constant and extrapolated pressure of left ventricular pressure decay in coronary artery disease. J Am Coll Cardiol 1985;6:597-02.

28. Lee MA, Dae MW, Langberg JJ, et al. Effects of long-term right ventricular apical pacing on left ventricular perfusion, innervation, function and histology. J Am Coll Cardiol. 1994;24:225-32.

29. Tse HF, Lau CP. Long-term effect of right ventricular pacing on myocardial perfusion and function. J Am Coll Cardiol 1997;29:744 49.
30. Lewis $\mathrm{CW}$, Owen $\mathrm{CH}$, Zipprich DA, et al. The effects of local ventricular pacing on recovery from regional myocardial ischemia. J Surg Res 1993;54:360-67.

31. Chen CC, Shen TY, Chang MC, et al. Stress-induced myocardial ischemia is associated with early post-stress left ventricular mechanical dyssynchrony as assessed by phase analysis of 201Tl gated SPECT myocardial perfusion imaging. Eur J Nucl Med Mol Imaging 2012;39:1904-09.

32. Hida S, Chikamori T, Tanaka H, et al. Diagnostic value of left ventricular dyssynchrony after exercise and at rest in the detection of multivessel coronary artery disease on single-photon emission computed tomography. Circ J 2012;76:1942-45.

33. Haugaa KH, Smedsrud MK, Steen T, et al. Mechanical dispersion assessed by myocardial strain in patients after myocardial infarction for risk prediction of ventricular arrhythmia. JACC Cardiovasc Imaging 2010;3:247-56.

34. Bugiardini R1, Estrada JL, Nikus K, et al. Gender bias in acute coronary syndromes. Curr Vasc Pharmacol 2010;8:276-84.

35. Berger JS, Elliott L, Gallup D, et al. Sex differences in mortality following acute coronary syndromes. JAMA 2009;302:874-82.

36. Berthillot C, Stephan D, Chauvin M, et al. In-hospital complications after invasive strategy for the management of Non STEMI: women fare as well as men. BMC Cardiovasc Disord 2010;10:31.

37. Berman DS, Kang X, Hayes SW, et al. Adenosine myocardial perfusion single-photon emission computed tomography in women compared with men. Impact of diabetes mellitus on incremental prognostic value and effect on patient management. J Am Coll Cardiol 2003;41:1125-33.

38. Hachamovitch R, Berman DS, Kiat $\mathrm{H}$, et al. Effective risk stratification using exercise myocardial perfusion SPECT in women: gender-related differences in prognostic nuclear testing. J Am Coll Cardiol 1996;28:34-4.

39. Cerci MS, Cerci JJ, Cerci RJ, et al. Myocardial perfusion imaging is a strong predictor of death in women. J Am Coll Cardiol Img 2011;4:880-88.

40. AlJaroudi W, Alraies MC, Hachamovitch R, et al. Association of left ventricular mechanical dyssynchrony with survival benefit from revascularization: a study of gated positron emission tomography in patients with ischemic LV dysfunction and narrow QRS. Eur J Nucl Med Mol Imaging 2012;39:1581-91.

41.AlJaroudi W, Alraies MC, Menon V, et al. Predictors and incremental prognostic value of left ventricular mechanical dyssynchrony response during stress-gated positron emission tomography in patients with ischemic cardiomyopathy. J Nucl Cardiol 2012;19:958-69.

42.Shin SH, Hung CL, Uno H. Mechanical dyssynchrony after myocardial infarction in patients with left ventricular dysfunction, heart failure, or both. Circulation 2010;121:1096-03. 